\title{
DIRECT SIMULATION OF REENTRY FLOWS WITH IONIZATION
}

\author{
Ann B. Carlson* \\ NASA Langley Research Center \\ H. A. Hassan** \\ North Carolina State University
}

\begin{abstract}
The Direct Simulation Monte Carlo (DSMC) method is applied in this paper to the study of rarefied, hypersonic, reentry flows. The assumptions and simplifications involved with the treatment of ionization, free electrons and the electric field are investigated. A new method is presented for the calculation of the electric field and handling of charged particles with DSMC. In addition, a two-step model for electron impact ionization is implemented. The flowfield representing a $10 \mathrm{~km} / \mathrm{sec}$ shock at an altitude of $65 \mathrm{~km}$ is calculated. The effects of the new modeling techniques on the calculation results are presented and discussed.
\end{abstract}

\section{Nomenclature}

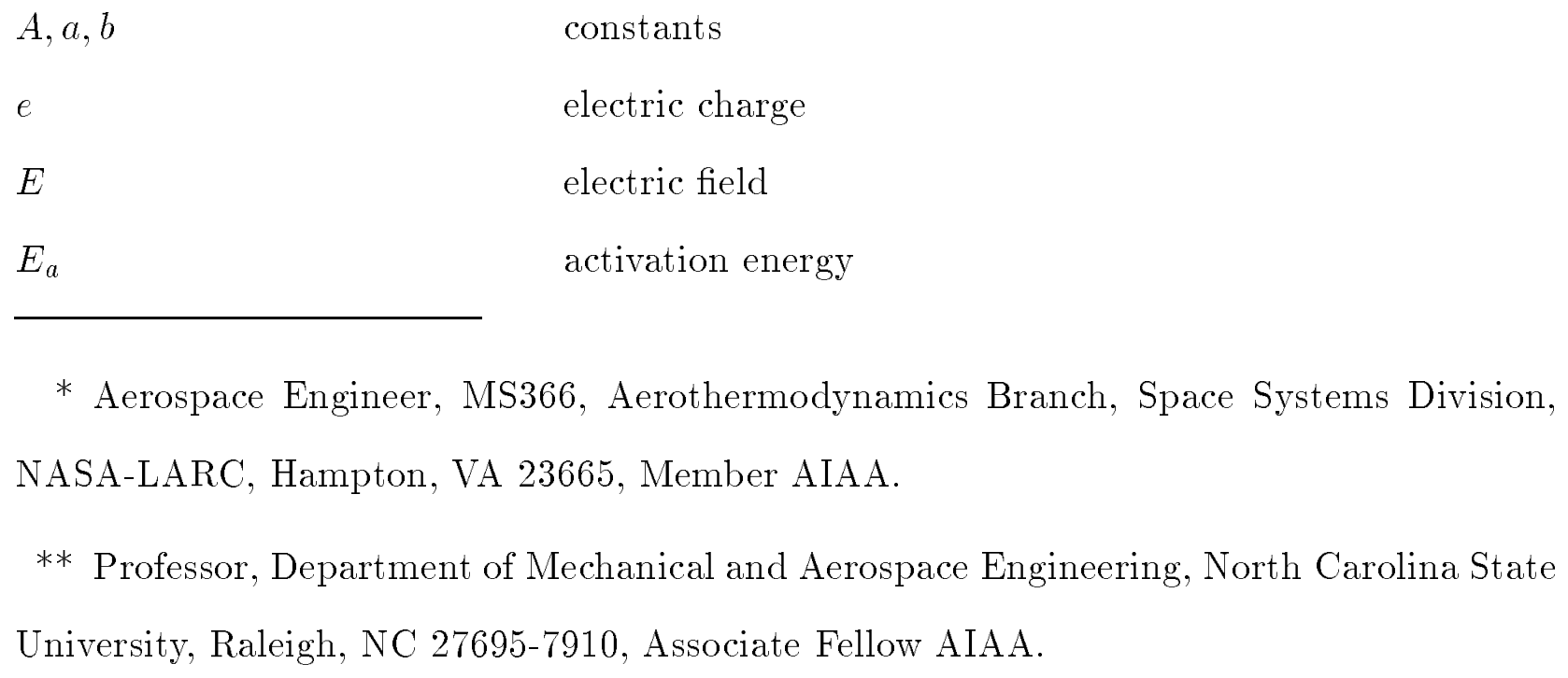

* Aerospace Engineer, MS366, Aerothermodynamics Branch, Space Systems Division, NASA-LARC, Hampton, VA 23665, Member AIAA.

** Professor, Department of Mechanical and Aerospace Engineering, North Carolina State University, Raleigh, NC 27695-7910, Associate Fellow AIAA. 


\begin{tabular}{ll}
$E_{c}$ & energy of colliding particles \\
$E_{\infty}$ & ionization energy \\
$k$ & Boltzmann constant \\
$k(T)$ & chemical rate coefficient \\
$m$ & mass \\
$\hat{n}$ & number density \\
$n$ & principal quantum number \\
$\mathcal{N}$ & nitrogen atom \\
$\mathcal{O}$ & oxygen atom \\
$P_{1}$ & energy of excited state \\
$P r$ & steric factor \\
$q$ & electric charge \\
$R$ & relaxation collision number \\
$R y$ & Rydberg constant \\
$t$ & time \\
$T$ & temperature \\
$\vec{v}$ & particle velocity \\
$x$ & distance coordinate \\
$\zeta$ & internal degrees of freedom \\
$\nu$ & collision frequency \\
$\sigma_{r}$ & reaction cross-section \\
& \\
\hline &
\end{tabular}

\section{Subscripts}

e

$h$

i

$s$ value for electrons

value for heavy particles

value for ions

value for particle $s$ 


\section{Superscript}

particle in excited state

\section{Introduction}

The Direct Simulation Monte Carlo (DSMC) method of Bird[1] has been used for more than two decades in the numerical simulation of physical fluid flows. The method has been developed to the extent that it can be successfully applied to typical engineering flows involving shocks in air with thermal and chemical nonequilibrium. Recent work has been focused on extending the method to treat flows with ionization and radiation[2,3]. This level of complexity is necessary to accurately predict the reentry flow fields for such vehicles as the Aeroassist Flight Experiment (AFE) and Aeroassisted Orbital Transfer Vehicles (AOTV).

Certain difficulties are encountered when using DSMC with flows involving ionization. The presence of ions and electrons in the flow leads to electric field effects such as charged particle acceleration and ambipolar diffusion. Free electrons in the flow have extremely high velocities and collision rates compared with the heavy particles. This implies considerably more computational time to accurately simulate these parameters. However, the determination of the free electron properties is particularly important because the radiation environment and level of electron impact ionization are dependent on the energy of the free electrons.

The method which has been employed by Bird[2] in previous simulations of flow with ionization involves some approximations which may introduce errors in the determination of free electron properties. In cases which require an accurate determination of these properties, an alternate method is proposed which involves the calculation of the ambipolar diffusion induced electric field and its influence on the motion of the charged particles. An alternative approach to the calculation of electron impact ionization is also introduced. This method involves the assumption that the reaction proceeds in two steps: electron impact excitation followed by ionization from the excited state. 


\section{The DSMC Method}

Statistical methods are used in the DSMC formulation to obtain a simulation of the flow of low density gases. The simultaneous computation of the trajectories of thousands of simulated molecules are performed in physical space. Collisions between the particles and interactions of the particles with the boundaries of the computational region are modeled. The flow properties are determined from the averages of the particle properties after a very large number of such interactions have taken place. A cell structure in the computational space is used to determine potential collision partners and for sampling of flow properties. The time parameter in the flow may be identified with real time and is advanced according to the collision frequency appropriate for the flow. An important assumption in the DSMC method is that the molecular motion and intermolecular collisions may be uncoupled over the small time step, $\Delta t$, used to advance the calculation. Thus, collisions between particles proceed until the time is advanced in the flow by $\Delta t$, then the particles are moved the distance determined by their current velocities and $\Delta t$. This has been shown[1] to be a valid assumption when the time step is less than the local average collision time

$$
\Delta t<1 / \nu
$$

where $\nu$ is the local average collision frequency. The position coordinates, velocity components and internal state of each molecule are stored in the computer and are modified with time as the molecules are followed through representative collisions and boundary interac-

tions. In addition to changes in translational and internal energy, collisions may result in chemical reactions or ionization. Particles enter or exit the flow at computational boundaries representing free stream, a vacuum, or a known flow solution. Advantage may be taken of flow symmetries to reduce the number of dimensions of the grid and the number of position coordinates that need to be stored, but the collisions are calculated as three-dimensional phenomena. Although the flow is always unsteady, boundary conditions may be such that a steady flow is obtained as the large time state of the unsteady flow. 
Models of the physical processes in the flow are required. The Variable Hard Sphere (VHS) model is used for the molecular interaction potential. The Borgnakke and Larsen phenomenological model is employed in the rotational and vibrational internal energy calculations. The implementation of these models is detailed in reference 1 . The nonequilibrium chemical reactions proceed on the basis of collision energy dependent steric factors or reaction cross-sections. Because data are not generally available for the reaction cross-sections, a form of the collision theory that is consistent with the VHS model[4] is used to convert temperature dependent rate constant data to collision energy dependent steric factors. The reaction rates are given in reference 5 .

For the calculations presented in this paper, a one dimensional DSMC stagnation streamline program is used. This program can be used to calculate a standing shock wave flowfield by adjusting the boundary conditions. Initially, a stagnation streamline calculation is performed where the boundary at one end is the undisturbed freestream while the other boundary is a stationary wall. When the shock reaches the desired position, molecular removal downstream of the shock commences. The molecular removal is conducted in such a way that mass, momentum and energy are conserved. It has been shown that this is achieved if the molecules are removed with a probability proportional to the square of their velocity component normal to the stream[6]. For the standing shock wave simulation, molecule removal is immediately adjacent to the wall and the remainder of the flow is exactly one dimensional.

\section{Modeling of Plasmas}

Several difficulties arise when the modeling of plasmas is attempted with DSMC. In general, the DSMC programs model only binary collisions in the flow. This is valid when three body collisions and multi-body charged particle interactions are insignificant in

comparison with the two-body collisions in the flow. For the hypersonic reentry flows of interest in this paper, multi-body charged particle collisions may be ignored if the ionization 
is less than $3-4 \%$. Charged particles are thus a minor species in the flow. Because each simulated particle represents an extremely large number of real particles, flow fluctuations in the simulation are many orders of magnitude larger than those in the real gas. These fluctuations in a charged gas could result in the prediction of an electric field which is far stronger than that in the real field. Also, the electron velocity and collision frequency are much higher than that for heavy particles. This implies a much smaller computational time step.

In early simulations[2], a simplified procedure for the modeling of plasmas was adopted. To prevent the diffusion of electrons out of the flow, each electron is associated with an ion at the time of its formation. Collisions are calculated for the electrons as for the heavy particles. However, they are moved in relation to the position of their associated ion rather than according to their velocities. This assures charge neutrality in the flow and eliminates the need to calculate complex electron trajectories. The time step used in the simulation is the one associated with molecular collisions rather than with the electron collision frequency. Because the movement of the electrons relative to the ions is restricted, the explicit evaluation of the electric field is not required. Experimental studies indicate that at high altitudes (above $70 \mathrm{~km}$ ) the effects of ambipolar diffusion are significant and should not be ignored[7]. Therefore, an iterative solution to account for this effect is used[6]. After obtaining a flow field solution, the electric field is estimated from a form of the Langmuir and Tonks[8] equation.

$$
E=\left(k T_{e} / e\right) d\left(\ln \left(\hat{n}_{e}\right)\right) / d x
$$

This electric field is then considered in calculating the movement of the ions and a new flowfield solution is obtained. The calculation procedure is repeated until the solution is converged.

A major problem with the above method is that the position of the electrons is artificially constrained (each electron associated with a specific ion). In the real flow, electrons from the higher temperature regions will diffuse into the lower temperature regions 
and vice versa. The use of the Langmuir and Tonks equation is questionable because it is a continuum formulation. The dependence of the equation on the gradient of the electron number density magnifies the errors associated with the statistical sampling of a minor species. Equation (2), which is the form of the equation used in the previous DSMC simulations, can be derived from the (continuum) momentum equations for the ions and electrons. Its derivation requires the following assumptions; slightly ionized gas, net current of zero, and constant electron temperature. If the electron temperature is not constant, then $E$ is proportional to the gradient of electron pressure and not to the electron density. Thus, Equation (2) is not valid in the shock region.

In view of these difficulties, a new method for handling plasmas with DSMC is proposed. The concept of ambipolar diffusion is used explicitly in the modeling to determine charged particle motion and the electric field. Ambipolar diffusion results when the lighter electrons tend to diffuse faster than the ions for flows involving a mass density gradient. A charge separation and resulting electric field are produced. The electric field retards the electron diffusion while enhancing the diffusion of the positive ions. The modeling proceeds as follows.

During the movement routine, the velocities of a charged particle in a given cell are determined from

$$
m_{s} d \overrightarrow{v_{s}} / d t=q_{s} \vec{E}
$$

The average velocity of each charged particle in a local region is written according to this equation. Then, the average velocity of ions and of electrons is determined by summing over the charged particles in that region. The resulting equations can be solved for the local electric field when the requirements of a net current of zero and charge neutrality are imposed. Details of this approach are given in the appendix. The present simulation accounts fully for both density and temperature gradient effects on the electric field.

The electric field calculations are performed in the domain of a supercell, which consists of several adjacent computational cells (typically about 10). The supercell must contain several hundred particles in order to obtain a reasonable sample of charged particles. In 
addition, the electric field result is time averaged before it is used in the calculation of charged particle motion. Because of the disparity between the collision frequencies of the electrons and the heavy particles, each is moved based on its own time step. This yields $\Delta t_{e} \ll \Delta t_{h}$ and electrons move more often than the heavy particles. The electric field, $\vec{E}$, is calculated after each electron movement. The result is smoothed over the heavy particle time step, $\Delta t_{h}$, and used for the next $\Delta t_{h}$ in the calculations of charged particle velocity. Once the flow has reached steady state, the electric field is calculated by averaging.

Charge neutrality must be enforced in the simulation because the Debye length is much smaller than any other characteristic length. In the present approach, the electrons and ions are not tied together and charge neutrality is not automatically ensured. The following procedure is used if, after the movement routine is exercised, the charge neutrality condition is not satisfied. Randomly selected electrons in supercells with excess electrons are moved to randomly chosen locations in neighboring supercells which have a deficiency of electrons until the number of electrons and ions in each supercell is equal.

\section{Electron Impact Ionization}

The flow fields considered in this study are sufficiently energetic that a significant contribution to the total ionization is from electron impact ionization reactions. The inclusion of these reactions in the DSMC program presents certain problems. The reactions have traditionally been handled as single step reactions, but the available rate constants for these reactions are not of a form which can be used directly with the DSMC methodology.

The continuum rate coefficients $k(T)$ for chemical reactions are specified by

$$
k(T)=a T^{b} \exp \left(-E_{a} /(k T)\right)
$$

In the DSMC method, these rate coefficients are used to determine collisional energy dependent steric factors. The steric factor, $P r$, is the ratio of the reaction cross-section 
to the total cross-section that results in the above rate coefficient. It is proportional to

$$
\operatorname{Pr} \propto\left(1-E_{a} / E_{c}\right)^{\zeta+b+1 / 2}, \quad E_{c} \geq E_{a}
$$

where $\zeta$ is a measure of the vibrational and rotational internal degrees of freedom which may contribute to the reaction. Because $\operatorname{Pr}$ is zero for $E_{c} \leq E_{a}$, the representation is valid as long as

$$
\zeta+b+1 / 2>0
$$

The values of $b$ given by Park and Menees[5] for the electron impact ionization impact reactions

$$
\begin{gathered}
\mathcal{O}+e \longrightarrow \mathcal{O}^{+}+e+e \\
\mathcal{N}+e \longrightarrow \mathcal{N}^{+}+e+e
\end{gathered}
$$

are -3.9 and -3.82 respectively. Because the reactants are monatomic gases, $\zeta=0$ and the criterion indicated by Eqn.(6) is violated. The method that was employed to produce the rates traditionally used for these reactions involves determining an average flow temperature and replacing $T^{b}$ in the rate coefficient by its value at that temperature. This, in effect, changes $k(T)$ to

$$
k(T)=A \exp \left(-E_{a} /(k T)\right), \quad A=a\left(T_{a v g}\right)^{b}
$$

In the DSMC simulations this is not accurate in the immediate vicinity of the shock because the temperature is changing rapidly and is considerably different from the average temperature used in the rate calculation.

An alternate method is proposed which uses the assumption that these two reactions proceed via a two-step chain involving excitation followed by ionization from the excited state. Thus, the ionization of atomic nitrogen proceeds by

$$
\begin{gathered}
\mathcal{N}+e \longrightarrow \mathcal{N}^{*}+e \\
\mathcal{N}^{*}+e \longrightarrow \mathcal{N}^{+}+e+e
\end{gathered}
$$


with similar reactions for atomic oxygen. In his paper on ionization in air behind high speed shock waves, Wilson[9] asserts that the rate limiting step in this ionization process is the excitation of nitrogen atoms to the $3 s^{4} P$ state and oxygen atoms to the $3 s^{5} S$ state. Experimental reaction cross-sections are available for these excitation processes from Stone and Zipf[10-11]. These cross-sections are used directly in the program when determining the probability of excitation after a collision.

The data show a much larger excitation cross-section for nitrogen than for oxygen for these two states. This suggests a higher rate of ionization for nitrogen, but the available data do not indicate that this is the case. An investigation of the processes involved reveals that the nitrogen $3 s^{4} P$ state has a short radiative lifetime while the oxygen $3 s^{5} S$ state is stable. Thus, the nitrogen may radiate from the excited state before it has a chance to ionize. To determine if the nitrogen atom radiates, a process similar to that used for rotational and vibrational transitions is employed. A relaxation collision number, $R$, is determined from the product of the radiative lifetime and the average collision frequency for nitrogen atoms at that point in the flow. The quantity $1 / R$ gives the probability of transition for a single collision. For extremely rarefied flows the value for $R$ may be less than 1 . This indicates that the radiative lifetime is less than the average time between collisions and the nitrogen atom has very little chance of ionizing by this mechanism. Therefore, this reaction is bypassed if the relaxation collision number is less than 1 . When the DSMC results using the onestep reaction rates for the same flow conditions are examined, it is found that the electron impact ionization of nitrogen occurs very rarely. Thus, no major discrepancy between the two methods is introduced by bypassing this reaction for very low density flowfields.

For ionization from the highly excited state, the quantum defect method[12] and the experimentally determined cross-section equation of Lotz[13] are used. For a single electron in the subshell and an impact electron energy near threshold, Lotz gives

$$
\sigma_{r}=a\left(E / P_{1}-1\right)(1-b) / P_{1}^{2}
$$


The values for the constants in the equation are determined in accordance with the assumption that the excited states are almost hydrogenic $\left(a=4 . \times 10^{-14} \mathrm{~cm} / \mathrm{sec}, b=.6\right)$. This is valid for all highly excited states of atoms. The quantum defect method requires the formula to be multiplied by a factor of $n^{4}$ where $n$ is the effective principal quantum number.

$$
n=\left(R y /\left(E_{\infty}-P_{1}\right)\right)^{1 / 2}
$$

Thus,

$$
\sigma_{r}=n^{4} a\left(E / P_{1}-1\right)(1-b) / P_{1}^{2}
$$

\section{Results}

Results are presented for the $10 \mathrm{~km} / \mathrm{sec}$ standing shock wave in air at .1 torr. This flowfield is of interest because it represents the conditions of an AVCO shock tube measurement[14]. It is also representative of the flows which might be experienced by the AFE. In the plots, the flow is from negative to positive $x$ with zero at the approximate shock center. For these conditions, the flowfield composition through the shock and the translational temperature profile are given in figures 1 and 2.

One of the major purposes of the paper is to show that the DSMC represents a selfconsistent framework and does not require borrowing any results from continuum theory in

order to describe slightly ionized gases. Thus, when the present results are compared with those of Reference 6 , the objective is not to pass judgement on the accuracy or suitability of the Langmuir-Tonks formala. We know it is inadequate when electron temperature gradients are important. Rather, it is to compare with the only other particle model that was used to calculate the flow under consideration.

The present method for the plasma calculations has a significant effect on the electron temperature through the shock (figure 3 ). The peak temperature is lower and occurs further upstream of the shock. Also, energetic electrons are present through a much larger portion 
of the flow. The sharp peak in electron temperature which is predicted with Bird's method is the result of limiting the electron mobility by requiring the charged particles to move only in electron-ion pairs. The broader distribution of energetic electrons seems more physically reasonable. The shift in the peak demonstrates the tendency of the energetic electrons to diffuse to the lower density portions of the flow.

The electric field for the two cases is shown in figure 4. The present method shows an electric field which is larger upstream of the shock than that which was predicted by the traditional method. This result agrees with the altered electron temperature distribution. The field is strongest where the electron velocity is largest. The difference in the magnitude of the electric field may result from the use of the continuum and constant electron temperature assumptions of the Langmuir-Tonks equation used in Bird's method.

The electron concentration is somewhat less for the present method than for the Bird's method (figure 5). This is probably because the peak electron temperature is lower and occurs further upstream where the heavy particle temperature is lower. As a result there is less energy available for reactions involving electron impact in this portion of the flow.

Implementing the two-step electron impact reaction model has some effect on the results for electron temperature in the immediate vicinity of the shock (figure 6). The two-step modeling allows more accurate matching of the experimental cross-sections and reaction rates in the high temperature gradient region. The electron density (figure 7) is a bit smaller at the center of the shock but increases in the region behind the shock. As can be seen from the concentration of nitrogen and oxygen ions (figures 8 and 9 ) the ionization of the atoms is increased in the region approximately .25 to $.5 \mathrm{~cm}$ behind the shock when the two-step modeling technique is used.

\section{Conclusions}

Some changes to the DSMC method for modeling charged particles and electric field effects have been introduced. These changes allow a more accurate representation 
of ambipolar diffusion effects and electron impact ionization reactions. The results are noticeably different for electron density and electron temperature in the flow. It is recommended that this method be used in future simulations when the determination of these properties is essential.

\section{Acknowledgement}

The authors would like to acknowledge many helpful comments and suggestions by Drs.

Carl D. Scott of the NASA Johnson Space Center and Graeme A. Bird of the University of Sydney, Australia. One of the authors ( $\mathrm{HAH}$ ) would like to acknowledge support by NASA's Cooperative Agreement NCCI-112, the Hypersonic Aerodynamic Program Grant NAGW-1022 funded jointly by NASA, AFOSR and ONR, and the Mars Mission Research Center funded by NASA Grant NAGW-1331.

\section{References}

1. Bird, G.A.,'Monte-Carlo Simulation in an Engineering Context', Progress in Astronautics and Aeronautics;Rarefied Gas Dynamics, Vol. 74, Part 1, edited by Sam S. Fisher, 1981, pp239-255.

2. Bird, G.A., 'Low Density Aerothermodynamics', AIAA Paper 85-0994, 1985.

3. Moss, J.N.; Bird, G.A.; and Dogra, V.K.; 'Nonequilibrium Thermal Radiation for an Aeroassist Flight Experiment Vehicle', AIAA Paper 88-0081, 1988.

4. Bird, G.A., 'Direct Simulation of Multi-Dimensional and Chemically Reacting Flows', Rarefied Gas Dynamics, edited by R. Campargue, CEA, Paris, 1979, pp365388.

5. Carlson, A.B.; Hassan, H.A.; and Moss, J.N.; 'Monte Carlo Simulation of Reentry Plasmas', AIAA Paper 89-0683, 1989.

6. Bird, G.A., 'Direct Simulation of Typical AOTV Entry Flows', AIAA Paper 86-1310, 1986. 
7. Schexnayder, C.J., Jr.; Huber, P.W.; and Evans, J.S.; 'Calculation of Electron Concentration for a Blunt Body at Orbital Speeds and Comparison With Experimental Data', NASA TN D-6294, 1971.

8. Langmuir, I. and Tonks, I.; Physics Reviews, Vol. 34, 1929, p874.

9. Wilson, J., 'Ionization Rate of Air Behind High Speed Shock Waves', Physics of Fluids, Vol. 9, No. 10, October 1966, pp1913-1921.

10. Stone, E.J., and Zipf, E.C., 'Excitation of Atomic Nitrogen by Electron Impact', Journal of Chemical Physics, Vol. 58, 1973, pp4278-4284.

11. Stone, E.J., and Zipf, E.C., 'Electron-Impact Excitation of the 3S and 5S States of Atomic Oxygen', Journal of Chemical Physics, Vol. 60, 1974, pp4237-4243.

12. Griem, H.R., Plasma Spectroscopy, McGraw-Hill Book Company, New York, 1964.

13. Lotz, C., 'Electron-Impact Ionization Cross-Sections and Ionization Rate Coefficients for Atoms and Ions from Hydrogen to Calcium', Zeitschrift fur Physik, Vol. 216, 1968, pp241-247.

14. Allen, R.A., Rose, P.H. and Camm, J.C.;'Nonequilibrium and Equilibrium Radiation at Supersatellite Reentry Velocities', AVCO-Everett Research Laboratory, Everett, MA, Res. Rep. 156, 1962. 


\section{Appendix: Calculation of Electric Field}

The instantaneous electric field in a given electric field cell (or supercell) is determined from the consideration that the net ion current is equal to the net electron current. The resulting quantity, like many others of interest such as heat flux, stress, etc., is given in terms of the instantaneous particle velocities. The mean electric field at a given point in the flowfield is determined by averaging over a large sample.

Replacing the electric field by its average value over the time step $\Delta t$, the average velocity of a charged particle during $\Delta t$ follows from equation (3) as

$$
\vec{v}_{s}=\vec{v}_{s, 0}+\left(q_{s} \vec{E} \Delta t / 2 m_{s}\right)
$$

where $\vec{v}_{s, 0}$ is the particle velocity at the beginning of the time step, $m_{s}$ is the particle mass and $q_{s}$ is the particle charge. The average ion velocity for that time step is

$$
\overrightarrow{V_{i}}=\frac{\sum \vec{v}_{\alpha}}{\sum N_{\alpha}}
$$

where $\alpha$ refers to an ion species and $N_{\alpha}$ is the number of particles of that species in the supercell. The average electron velocity during the time step is

$$
\vec{V}_{e}=\sum \vec{v}_{e, 0} / N_{e}-\left(e \vec{E} / m_{e}\right)(\Delta t / 2)
$$

where $e$ is the charge of the electron. Because charge neutrality is required in the supercell, $N_{e}=\sum N_{\alpha}$, assuming singly ionized ions. The electric field is calculated by setting $\vec{V}_{i}=\vec{V}_{e}$ and solving the resulting equation. The result can be written as

$$
e \vec{E} \Delta t / 2=\frac{\left[\sum \vec{v}_{e, 0}-\sum \vec{v}_{\alpha, 0}\right] / N_{e}}{\sum 1 / m_{\alpha}+1 / m_{e}} \simeq m_{e}\left[\sum \vec{v}_{e, 0}-\sum \vec{v}_{\alpha, 0}\right] / N_{e}
$$

The above formula for the instantaneous electric field is averaged over many samples to give the mean electric field. 


\section{Captions}

Fig. 1 Composition through $10 \mathrm{~km} / \mathrm{s}$ shock in air at .1 torr

Fig. 2 Translational temperature through the shock

Fig. 3 Electron temperature through the shock

Fig. 4 Electric field through the shock

Fig. 5 Electron concentration through the shock

Fig. 6 Electron temperature through the shock

Fig. 7 Electron concentration through the shock

Fig. $8 N^{+}$concentration through the shock

Fig. $9 \mathrm{O}^{+}$concentration through the shock 